\title{
Philosophiques
}

\section{Christian Saint-Germain, Écrire sur la nuit blanche : l'éthique du livre chez Emmanuel Lévinas et Edmond Jabès, Montréal, Presses de l’Université du Québec, 1992, 290 pages.}

\section{Pascale Quiviger}

Volume 21, numéro 2, automne 1994

Les femmes et la société nouvelle

URI : https://id.erudit.org/iderudit/027301ar

DOI : https://doi.org/10.7202/027301ar

Aller au sommaire du numéro

Éditeur(s)

Société de philosophie du Québec

ISSN

0316-2923 (imprimé)

1492-1391 (numérique)

Découvrir la revue

Citer ce compte rendu

Quiviger, P. (1994). Compte rendu de [Christian Saint-Germain, Écrire sur la nuit blanche : l'éthique du livre chez Emmanuel Lévinas et Edmond Jabès,

Montréal, Presses de l'Université du Québec, 1992, 290 pages.] Philosophiques, 21(2), 627-631. https://doi.org/10.7202/027301ar d'utilisation que vous pouvez consulter en ligne. 
Christian Saint-Germain, Ecrire sur la nuit blanche: l'éthique du livre chez Emmanuel Lévinas et Edmond Jabès, Montréal, Presses de l'Université du Québec, 1992, 290 pages.

\section{par Pascale Quiviger}

Christian Saint-Germain, professeur de sciences religieuses à l'Université du Québec à Montréal, nous livre ici le texte ardu de sa thèse de doctorat. Il s'agit d'une réflexion « logopériphérique », s'inscrivant dans la lignée contemporaine du renoncement à l'unité d'une conscience maîtresse d'elle-même, tributaire de la perte du fondement qui renvoie ce qui reste du sujet à la finitude et le condamne au nomadisme.

La thèse que veut soutenir l'A. se base sur deux postulats : d'une part, le rapport avec l'altérité de l'Autre et l'écriture sont liés par une même absence et par une même recherche de ce qui s'absente; d'autre part, « l'écriture de l'éthique a pour tâche l'élaboration d'une éthique de l'écriture » (p. xx). Lévinas et Jabès sont pris à témoin d'une telle recherche, à travers et par le livre, de ce qui se dérobe à la présence : recherche du Livre premier chez Jabès, recherche de l' Autre chez Lévinas. Plus globalement, la «judaïté » ellemême tient lieu de « modèle de déportation de lecture » (p. xxir), déportation géographique d'un peuple nomade, et liaison essentielle au Livre, Livre de la Loi du Tout-Autre. Pour Lévinas, comme pour Jabès, comme pour le juif, le délogement du Même ne serait soutenu que par un rapport au livre dans lequel l'écriture congédie toute ontologie pour renvoyer à un fond sans fond.

Il ne s'agit pas uniquement de maintenir que le livre est affrontement du plus difficile, « geste d'appartenance à tout ce qui transite » (p. xxx), mais bien que par le livre, et seulement par lui, s'effectue la sortie du Même et s'inaugure le rapport d'altérité : « L'éthique commence au moment oủ la différence d'autrui, 
mise en question, intrigue par l'écriture le moi » (p. 163). Cette thèse, qui se veut donc proprement éthique, se situe, par rapport aux théories contemporaines, dans un refus conjoint de toute éthique circonstancielle (trop empirique) et de toute pensée de l'être (trop éthérée); elle se propose plutôt de montrer comment l'écriture fait surgir les problèmes éthiques et comment les problèmes éthiques se ramènent, en dernière instance, au problème de l'écriture. Ainsi, « il importe de montrer que dans la modernité, seul le livre peut, à travers l'humilité d'un rapport de lecture, remettre en perspective un lien éthique à autrui $\gg$ (p. xI). Seule la question du livre constitue une remise en question de l'autonomie de la conscience. Seule la lecture est « ouverture préalable à l'ouverture à autrui » (p. 25), dans la mesure où elle révèle que l'Autre habite toujours-déjà le même.

Le trajet emprunté par l'A. passe d'abord par l'œuvre de Jabès (quatre premiers chapitres), chez qui il tente de mettre en évidence l'irréductible distance entre le Livre de Dieu et le livre de l'homme, distance par laquelle l'homme se voit rendu à une liberté qui est aussi douloureuse séparation. Dans le livre se répète sans cesse cette rupture d'avec l'origine, l'entrée dans la nuit qui sauve de la nuit, la vertigineuse rencontre du vide, l'emprunt d'un nom toujours endetté en regard du Nom, la perte d'une identité propre, d'une filiation, le retard constitutif de la conscience, le renoncement à l'apaisement du désir, à l'accès au visage de Dieu, au visage de l'autre, à la connaissance de la Loi. Le livre jabésien donne Dieu pour le prototype de la disparition, voire de la mort: dans l'impossibilité où se trouve l'homme de voir le visage de Dieu et de le représenter, se révèle l'impossibilité pour l'homme de connaître son propre visage. L'espace d'écriture, entre-deux du Livre et du livre, ne peut qu'être lieu où se défait l'écriture à mesure qu'elle se fait, lieu de biffure, de rature, de dé-nomination. Le livre ne se peut que comme passage, attente, mouvement, incantation. Le rapport du livre au Livre et le rapport du moi à Autrui, comme « renoncement à l'autorité de la parole pleine » (p. 52), mettent profondément en question la pensée elle-même et s'attirent les foudres de la philosophie.

L'A. ne manque pas ici de développer plusieurs thèmes d'ordre psychanalytique : question de la différence sexuelle, tentative de surpasser le père, d'échapper au corps omniprésent de la mère, de découvrir le sens et l'origine de son nom (non), symbolique de la coupure, fantasme originaire de s'affranchir de la Loi et du Livre qui devient surmoi exacerbé. Écrire serait ainsi un effort identitaire, l'élaboration d'une question au sujet de soi-même, question supposément non narcissique (p. 57) dans la mesure où elle aboutit inévitablement sur l'Autre que soi. Seule l'écriture se ferait à la fois recherche obstinée du premier objet de désir et mise au jour de l'absence de cet objet; seul l'écrivain serait à même d'assumer l'absence que toute autre activité ne vise qu'à dissimuler.

Avant de passer à l'étude des textes lévinassiens, l'A. s'attarde à une définition approximative du point de rencontre entre «l'éthique de l'écriture » chez Jabès et l'« écriture de l'éthique » chez Lévinas, en présentant ce point comme « un même point d'horreur » (p. I64), une même obsession, un même « déchirement de la conscience » (p. i62), bien que ni ce point, ni cette obsession, ni ce déchirement ne se donnent dans les faits un même objet, ce qui concerne le Livre chez Jabès concernant l'Autre chez Lévinas. 
Les trois derniers chapitres sont donc consacrés à l'étude de Lévinas. L'auteur met en relief certaines des ambiguïtés du discours lévinassien : un sujet qui n'est pas un sujet, une passivité qui ne s'oppose pas à l'activité, une maternité au-delà des genres. Il montre comment le parcours lévinassien tend vers la corporéité (occasion de poser à nouveau la question de la différence sexuelle, cette fois mise en rapport avec la symbolique de la circoncision). Il insiste sur le débordement de la philosophie que provoque Lévinas à même le discours philosophique, disant en langue grecque ce qui excède la sagesse grecque.

Dans le dernier chapitre, l'A. s'attarde assez longuement sur la métaphore du féminin développée par Lévinas et il en montre bien l'équivocité. Il distingue à cet effet la métaphore très positive de la maternité, qui signifie la dénucléation de la subjectivité, de celle, plutôt péjorative, de la féminité, en tant que possibilité du mensonge, de l'ombre, de l'impudeur et de l'impureté. L'A. tente de discerner ce qu'un tel traitement du féminin doit à la tradition juive et à la tradition romanesque tout en tentant d'appliquer au discours de Lévinas lui-même les catégories qu'il déploie. L'écriture lévinassienne serait féminine, dans la mesure où elle met à nu la conscience, dans la mesure également où elle ouvre une blessure irrefermable et se tient en retrait, dans l'ombre d'une équivoque indissoluble.

En traitant de la métaphore du féminin, l'A. s'attaque certainement à l'une des parties les moins résolues de l'œuvre lévinassienne. Si son analyse ouvre de nouvelles pistes de lectures et pose de bonnes questions, on ne saurait pourtant considérer qu'il éclaire la situation, son discours faisant fi de l'ordre chronologique des textes sur lesquels il s'appuie et passant sans avertir du propre au figuré (de la femme à la métaphore de la femme, en passant par l'application de cette métaphore au texte lévinassien), donnant lieu à une confusion qui ne peut manquer de mettre mal à l'aise toute ... lectrice.

Par ailleurs, derrière l'ensemble de cette analyse du texte lévinassien se tient comme en attente l'hypothèse de fond de l' $\mathrm{A}$. qui veut que l'écriture constitue, pour Lévinas, « une préoccupation majeure », sinon la principale préoccupation. L'altérité dont traite Lévinas exige une rupture du texte et de la thématisation, un discours obsessif, répétitif, métaphorique qui, selon l'A., ne peut que fondre le contenu au contenant et faire ainsi du discours habité par l'Autre une altérité fondamentale du discours lui-même : ainsi, « seul le livre soutiendrait [...] un accès à autrui vécu comme différent » (p. 175). S'il est évident que le style de Lévinas ne peut être que profondément tributaire de l'ébranlement de la conscience dont il fait état, si sa volonté d'esquisser, à même le tissu du texte philosophique, ce qui ne peut que déchirer le texte, l'identification de la préoccupation pour l'Autre et de la préoccupation pour l'écriture ne me semble permise que par un flottement terminologique à demi sophistique. Plus encore, l'hypothèse d'une primauté de la question de l'écriture chez Lévinas me semble infidèle non seulement au texte lévinassien lui-même, mais encore à l'éthique dont il se réclame. Lévinas, par exemple dans Totalité et infini, insiste longuement sur la préséance du langage oral par rapport au langage écrit, la possibilité et l'essentiel du langage résidant dans l'adresse à un interlocuteur et dans la réponse à son appel, bien plus que dans le discours livresque. De la même manière, l'excellence qu'accorde Lévinas dans son œuvre 
tardive à tout texte qui se laisse habiter d'un souffle prophétique, se tenant en cela dans le sillage de la Bible, n'en renvoie pas moins à l'éthique interhumaine comme à sa revendication fondamentale et non son dérivé.

La trajectoire de Lévinas tend à mon avis vers la corporéité précisément pour qu'on n'oublie pas que c'est un autre de chair et de sang qui commande et appelle la subjectivité, que c'est du pain qu'il demande et que c'est de la mort dont il faut le garder. De même, c'est à partir de sa jouissance dans un monde économique que la subjectivité ouvre la porte de sa demeure (Totalité et infini), c'est dans son corps qu'elle se trouve affectée par autrui (Autrement qu'être); «Un corps animé ou une identité incarnée est la signifiance de cette non-indifférence » (Autrement qu'être, p. i14). En aucun cas l'éthique lévinassienne ne se détourne de cette chair et de ce sang pour se préoccuper d'une vague éthique de l'écriture, absorbée par la lettre ou dévoyée dans le symbolisme psychanalytique (par rapport auquel, d'ailleurs, Lévinas n'a jamais relâché sa méfiance). Autrui n'est « rapport au livre » (p. 23) que dans la mesure où le Livre, la Bible, renvoie les hommes à la solidarité. Car l'Autre ne se trouve pas d'abord dans la lettre : il est face à moi, et dans ce face-à-face réside la vocation la plus difficile de l'humanité. Les formules de Lévinas sont sans équivoque, par exemple : «Une relation avec le Transcendant [...] est une relation sociale » (Totalité et infini, p. 76); « Autrui m'importe dans toute sa misère matérielle » (Emmanuel Lévinas, Qui êtes-vous?, p. 99).

De surcroît, il semble que pour l'A., la subjectivité ne rencontre l'altérité dans l'écriture qu'au cours d'une recherche de sa propre origine et que, dans cette rencontre, il faille sauver le moi plus que l'autre : par exemple, « toute pensée, quelle qu'elle soit, s'oriente dans l'écriture comme un prodigieux effort pour s'accrocher à autrui, pour s'y aggripper et se tenir au-dessus du gouffre » (p. xxvi). Il m'apparaît au contraire que toute l'éthique lévinassienne se situe en marge d'une telle quête subjective dont l'autre rompt la légitimité. Autrui ne me renvoie à mon unicité qu'en tant qu'il m'assigne à « une responsabilité totale, qui répond de tous les autres et de tout chez les autres, même de leur responsabilité » (Ethique et infini, p. 95).

En somme, la thèse de l' A., si elle est en soi intéressante et assez originale, aurait ainsi gagné à se fonder sur elle-même plutôt que sur une œuvre qui lui soit rébarbative.

Par ailleurs, ce troisième auteur qui point, comme un spectre, derrière Jabès et Lévinas, ce troisième obsédé, le juif qui sanctionne le rapport essentiel à la Loi et à l'errance, m'apparaît ici trop hâtivement pris à témoin de la primauté du « geste d'écriture ». Prétendre que « la judaïté est inséparable de l'acte d'écrire » (p. 83) et qu'« être juif » constitue le « sort commun imparti à l'écrivain » (p. 133), m'apparaît téméraire et, peut-être même, indiscret à l'égard du judaïsme. N'est pas juif qui veut. L'identification de l'écrivain au juif n'est rendue possible qu'au prix (très élevé) d'une réduction de la « judaïté » à la pauvreté d'un concept ou d'une métaphore aisément manipulable.

Une conceptualisation semblable se retrouve d'ailleurs à bien des plans dans cet ouvrage, le plus souvent empruntée à d'autres auteurs et dépourvue de sens explicite : le Livre, l' absence, la Loi, le désir, la mort, le Nom, l'écrivain, Dieu, autant de « personnages » mis en scène dans un texte vague, torturé, difficile, truffé de citations et hachuré de notes. S'il est légitime et même 
nécessaire que le style se plie au propos, l'A. surestime de toute évidence la patience du lecteur en donnant dans un hermétisme à la limite du pédantisme, derrière lequel on ne peut s'empêcher de soupçonner un élément inavoué, inavouable, mais lequel ? Absence de contenu ou de franchise, complaisance ludique dans l'érudition? Ou, peut-être, comme il en mentionne lui-même la possibilité, désir propre à l'écrivain de semer le lecteur : « Écrire serait-il souhait inavouable que la lettre résiste, que ce qui s'écrit pour l'Autre ne soit jamais lu en totalité, discrétion, passion de ce qui ne peut se passer qu'à l'insu du lecteur $[. ..] \gg$ (p. 66) ?

Finalement, il faut bien le dire, le discours sur l'absence de fond du discours, discours lassant de la lassitude, devient ici fort irritant: l'hégémonie du « geste d'écriture », l'interminable ressassement du malheur et du vide, en repliant la lettre sur elle-même, lui ôtent toute la possible fécondité d'un rapport avec ce qui n'est pas elle et courent le risque combien néfaste d'encourager, sous des dehors d'altérité, de lucidité et de condamnation des idoles, la figure mythique de l'écrivain comme unique lieu de rencontre avec l'Innommable et condition de possibilité de l'émergence d'une véritable éthique interhumaine. Au détriment de ce qui se trouve en dehors du livre.

Département de philosophie

Université de Montréal 